\title{
Quasiparticle energy bands and Fermi surfaces of monolayer $\mathrm{NbSe}_{2}$
}

\author{
Sejoong Kim ${ }^{1,2, \text { f }}$ and Young-Woo Son ${ }^{1, \dagger}$ \\ ${ }^{1}$ Korea Institute for Advanced Study, Hoegiro 85, Seoul 02455, Korea \\ ${ }^{2}$ University of Science and Technology, Gajeong-ro 217, Daejeon 34113, Korea
}

(Dated: September 15, 2017)

\begin{abstract}
A quasiparticle band structure of a single layer $2 H-\mathrm{NbSe}_{2}$ is reported by using first-principles $G W$ calculation. We show that a self-energy correction increases the width of a partially occupied band and alters its Fermi surface shape when comparing those using conventional mean-field calculation methods. Owing to a broken inversion symmetry in the trigonal prismatic single layer structure, the spin-orbit interaction is included and its impact on the Fermi surface and quasiparticle energy bands are discussed. We also calculate the doping dependent static susceptibilities from the band structures obtained by the mean-field calculation as well as $G W$ calculation with and without spinorbit interactions. A complete tight-binding model is constructed within the three-band third nearest neighbour hoppings and is shown to reproduce our $G W$ quasiparticle energy bands and Fermi surface very well. Considering variations of the Fermi surface shapes depending on self-energy corrections and spin-orbit interactions, we discuss the formations of charge density wave (CDW) with different dielectric environments and their implications on recent controversial experimental results on CDW transition temperatures.
\end{abstract}

\section{INTRODUCTION}

After the first experimental success in isolating various single layers from layered materials such as graphite, hexagonal boron nitrides, transition metal dichalcogenides (TMD) and high-temperature cuprate superconductors $^{1}$, there have been tremendous efforts to understand physical properties of two-dimensional (2D) crystals 6 . Notably, owing to the reduced spatial dimension compared to their bulk counterparts, the Coulomb interaction in the $2 \mathrm{D}$ crystals as well as screening of substrates on which they are placed have played very important roles in modifying their electronic structures $3|5| 7 \mid 11$. For example, it is now well established that many-body interactions alter low-energy bands in graphene significantly 5112 17. Moreover, the substrate screening also changes the nature of Coulomb interactions in graphene on top of either dielectric materials or metals and hence modifies the bands further 13118 .

Besides graphene, several recent experiments have revealed new interesting physical properties in a monoand a few-layer TMDs such as series of phase transitions and novel superconductivity, being different from those shown in their bulk forms ${ }^{19}$ 31. Formation of charge density waves (CDWs) in three-dimensional metallic TMDs has attracted interests for the last couple of decades and origins of CDWs in some materials are still not settled yet ${ }^{32133}$. Therefore, the current efforts in investigating physical properties of thin flakes of TMDs may shed light on the origin of CDW phase in three dimensional TMDs and open a way to find the characteristic new collective phenomena in $2 \mathrm{D}$ crystals 2428 .

Among metallic TMDs, the stacked trigonal prismatic structure of niobium diselenide $\left(2 \mathrm{H}-\mathrm{NbSe}_{2}\right)$ is one of the most studied materials and an ideal system to study phase transitions as functions of temperature and dopings. It has been known for a long time ${ }^{32 \mid 33}$ that threedimensional stacking structure of $2 \mathrm{H}-\mathrm{NbSe}_{2}$ is metallic at room temperature and undergoes a CDW transition at $33 \mathrm{~K}$ before becoming a superconductor ${ }^{34 / 35}$ at 7.2 $\mathrm{K}$ although there has been the controversy regarding on the origin of CDW and the competition between CDW and superconducting (SC) states $32[33 / 36 / 45$. After a few earlier attempts to investigate physical properties of its thin flakes146/47, a couple of recent works have reported successful isolations of its single layer form on top of various substrates and measure their CDW and SC phase transitions 27 . 31 .

While all experiments $\sqrt[29]{3146 / 47}$ hitherto have shown that the superconducting transition temperature decreases but does not diminish completely when the thickness of $2 \mathrm{H}-\mathrm{NbSe}_{2}$ decreases to a single layer limit, the transition temperature $\left(T_{\mathrm{CDW}}\right)$ from metal to $\mathrm{CDW}$ phase differs from each other significantly ${ }^{30 \mid 31}$. The work by Xi et al $\stackrel{[30}{n}$ measured $T_{\mathrm{CDW}}$ of $145 \mathrm{~K}$, more than four times larger than the bulk $T_{\mathrm{CDW}}$ of $33 \mathrm{~K}$ whereas the work by Ugeda et al. reported that $T_{\mathrm{CDW}}$ is similar to or less than that of the bulk. Moreover, the former attribute the strong coupling mechanism to the formation of CDW at the very high temperature while the latter measured a small CDW energy gap of $4 \mathrm{meV}$ together with CDW modulation under high biases pointing to the puzzling dual nature (strong and weak) of CDW formation. It is noticeable that the former measured the transition in a sample on top of silicon substrate while the latter on top of epitaxial bilayer graphene (BLG) grown on $6 \mathrm{H}$ $\mathrm{SiC}(0001)$ surface.

Previous theoretical studies have shown that the bulk CDW phase of $2 \mathrm{H}-\mathrm{NbSe}_{2}$ does not have a prominent sharp peak at the specific CDW wave vector, rather showing a broad peak in the real part of the bare susceptibility and its imaginary part does not peak at the CDW wave vector at all $\underline{48}\left[\frac{50}{4}\right.$. These suggest a possible strong electron-phonon coupling mechanism to form the CDW phase 40 , ruling out the Fermi surface nesting mechanism 36 , 39 , or the saddle-point singularity driven 
CDW phase $\mathrm{C}^{5152}$. A recent first-principles calculation using a semi-local exchange-correlation functional predicts the CDW instability in the single layer structure with an enhancement of the electron-phonon interaction at a specific CDW wave vector differing from that of the bulk ${ }^{53}$. However, the predicted $4 \times 1 \mathrm{CDW}$ state is inconsistent with the recent measurement showing $3 \times 3 \mathrm{CDW}$ state by Ugeda and coworkers 31 . Another recent calculation using the similar method also suggests a good metallic behavior for a monolayer undistorted structure ${ }^{54}$ while a transport measurement shows a semimetallic nature ${ }^{1}$.

Considering significant changes in the low energy bands of the semimetallic and semiconducting $2 \mathrm{D}$ crystals with a proper inclusion of electronic self-energy correction as well as its modification by the substrate screening $5 / 7 / 10 / 12$ 18, a plain mean field calculation is not sufficient and it is necessary to investigate effects of suitable corrections from the many-body Coulomb interactions on the low energy bands of monolayer $\mathrm{NbSe}_{2}$. Although the CDW formation is quite sensitive to a shape of Fermi surface, its variation with the self energy corrections and alternations by the substrate screening have not been investigated yet fully. In this work, motivated by recent rapid progress in this field and experiments reporting different CDW formations $30 \mid 31$, we report first-principles density functional calculation and $G W$ approximation results of single layer $\mathrm{NbSe}_{2}$ in its normal metallic state which provide comprehensive pictures of the low energy electronic structures, a prerequiste to understand the controversal CDW features.

This paper is organized as follows. In Sec. II] we introduce a model system and calculation details. In Sec. III, our calculation results for the low energy band structures based on mean-field calculation methods and $G W$ approximation with and without spin-orbit interactions are presented. The doping dependent variations of Fermi surfaces are also discussed within various levels of approximations. The bare susceptibilities with calculated energy band structures are also presented in this section. Conclusions and discussion on CDW formation mechanism in the single layer are in Sec. IV] In addition, the effects of including semi core orbitals, detalied derivation of atomic SOCs in this system, constructions of a tight binding Hamiltonian within the three-band third nearest neighbor hopping between $d$-oribital of niobiums fit for the DFT-GGA and $G W$ energy bands, respectively, and discussions on $G W$ energy bands of the bulk $2 \mathrm{H}-\mathrm{NbSe}_{2}$ are presented in Appendix.

\section{SYSTEMS AND CALCULATION DETAILS}

Figure 1 displays the crystal structure of a single layer of $2 \mathrm{H}-\mathrm{NbSe}_{2}$. The single layer is composed of three sublayers: a triangular lattice of $\mathrm{Nb}$ at the center, which is sandwiched by two outermost layers of Se triangular lattice, forming a trigonal prismatic structure. The two Se sub-layers of the $2 H$ structure are mirror symmetric (a)

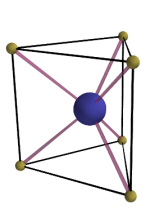

(b)

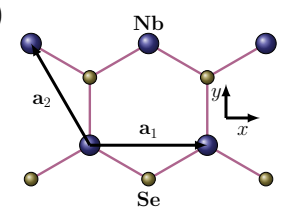

$(c)$

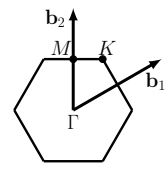

FIG. 1. (Color online) Crystal structure and Brillouin zone of a single layer of $2 \mathrm{H}-\mathrm{NbSe}_{2}$. (a) $2 \mathrm{H}$ structure of $\mathrm{NbSe}_{2}$. A transition metal atom ( $\mathrm{Nb}$, blue sphere) are surrounded by six chalcogens (Se, yellow sphere). Three of them lie on the upper plane, and the other three on the lower one. (b) Crystal structure from a top view. Primitive lattice vectors $\mathbf{a}_{1}$ and $\mathbf{a}_{2}$ are denoted. (c) The first Brillouin zone and reciprocal lattice vectors $\mathbf{b}_{1}$ and $\mathbf{b}_{2}$.

to each other with respect to the plane of the $\mathrm{Nb}$ layer as shown in Fig. 11(a). From a top view, the single layer of $2 \mathrm{H}-\mathrm{NbSe}_{2}$ looks like a hexagonal lattice.

We simulate the single layer of $2 \mathrm{H}-\mathrm{NbSe}_{2}$ by taking a vacuum layer of $12 \AA$. We increase the vacuum layer up to $20 \AA$ and find no changes in calculation results. Our relaxation calculation gives that the lattice constant of the layer is $a=3.45 \AA$ and the distance between $\mathrm{Nb}$ and Se sublayers is $1.68 \AA$, agreeing well with the previous studies 53154 .

We performed density functional theory (DFT) calculation by adopting the PBE generalized gradient approximation (GGA) $)^{55}$ for the exchange-correlation functional and the norm-conserving pseudopotential ${ }^{\sqrt{56}}$ with a nonlinear core correction 57 . Considering a crucial role of core levels in estimating self energy $\sqrt{58159}$, we treated the semicore $4 s^{2} 4 p^{6}$ electrons of $\mathrm{Nb}$ atoms as valence electrons and discuss its impact on the quasiparticle energy bands in Fig. 11 in Appendix A. We used the plane-wave DFT code QUANTUM-ESPRESSC ${ }^{60}$ with a cutoff of 55 Ry, a $40 \times 40 \times 1 k$-point grid, and a smearing temperature $k_{B} \tau=0.005$ Ry. Quasiparticle energies were calculated within the level of $G_{0} W_{0}$ approximation ${ }^{6162}$ implemented in the BERKELEYGW $\operatorname{code}^{\sqrt{63}}$ (hereafter, we will call the quasiparticle bands from $G_{0} W_{0}$ approximation as $G W$ energy bands for convenience). The slab truncation scheme was used to treat the Coulomb interaction for the single layer geometry 63 . We used unoccupied bands up to 5 Ry above the Fermi energy for the dielectric function calculation to achieve the convergence of calculations.

\section{RESULTS}

\section{A. Band Structure}

Figure 2 shows electronic band structures calculated by DFT-GGA (red dashed line) and $G W$ approximation (blue solid line) respectively along symmetric lines of the irreducible Brillouin zone (IBZ). Our DFT-GGA band structure shows a quite good agreement with previous 


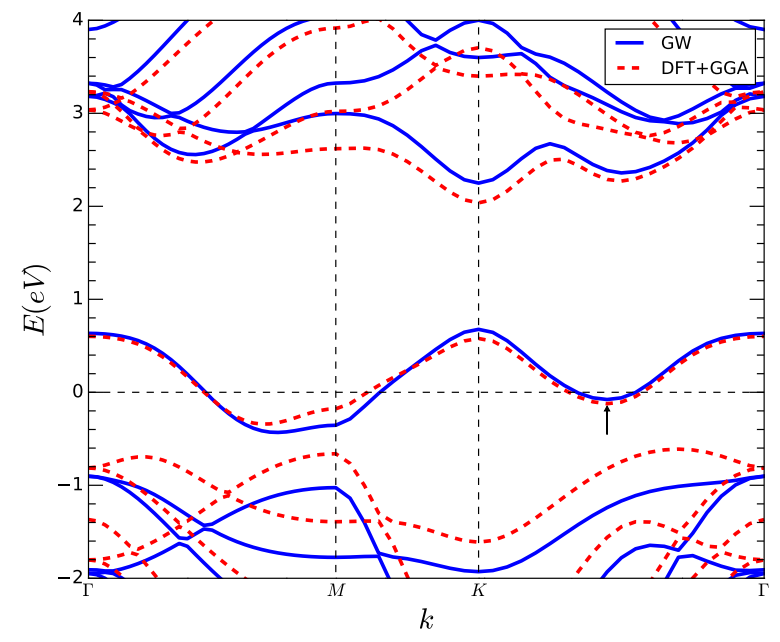

FIG. 2. (Color online) Electronic band structures of monolayer $\mathrm{NbSe}_{2}$ along the IBZ boundary. Red solid line and blue dashed one represent DFT-GGA and $G W$ band structures, respectively. The Fermi energy is set to be zero. Black arrow indicates the saddle point of the partially occupied band.

studies 53154 . We note that there are noticeable differences in a partially occupied band (up to spin degeneracy) around the Fermi level in the two calculation schemes. First, the energy band minimum, which is located in the middle of the $\Gamma M$ line in the DFT-GGA calculation, moves toward the $M$ point and becomes to be lowered, when the $G W$ correction is included. Second, the saddle point of the $G W$ band on the $\Gamma K$ line (indicated by black arrow in Fig. 2 is closer to the Fermi level than that of DFT-GGA. It is still slightly lower $(-77 \mathrm{meV})$ than the Fermi level $\left(E_{F}\right)$ while the DFT-GGA value for the point is much lower $(-123 \mathrm{meV})$ from the $E_{F}$. This close proximity of the saddle point to the charge neutral point is important in discussing CDW phase later. The shape of unoccupied DFT-GGA band structure and their $G W$ corrections shown in Fig. 2 looks quite similar to those of $\mathrm{MoS}_{2}{ }^{10}$ although the semiconducting $\mathrm{MoS}_{2}$ has a significant band gap enhancement from the selfenergy corrections 10 . Here, the spectral gap between the partially occupied band and unoccupied bands does not increases with the $G W$ correction but the band width of partially occupied band crossing the $E_{F}$ is enlarged by $17 \%$ with $G W$ approximations.

\section{B. Fermi Surface}

The Fermi surface also changes when the $G W$ correction is added to the DFT-GGA result. As shown in Fig. 3, calculated Fermi surfaces using the two methods basically contain two distinct hole pockets at the $K$ and $\Gamma$-points respectively. In the DFT-GGA Fermi surface shown in Fig. 3 (a), a hexagonal hole pocket is at the $\Gamma$-point and a rounded triangular hole pocket at the $K$-point agreeing
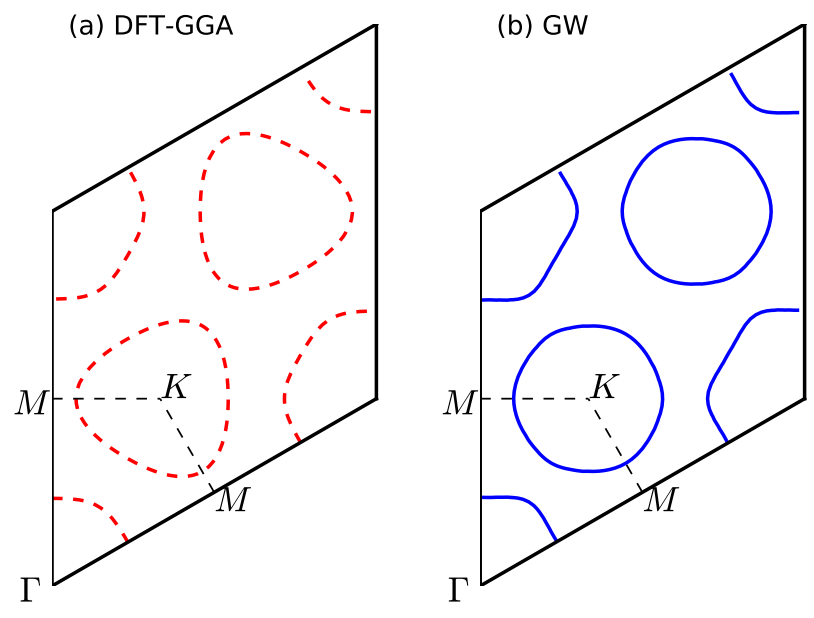

FIG. 3. (Color online) Fermi surfaces of DFT-GGA (red solid line) (a) and $G W$ (blue dashed line) energy bands (b).

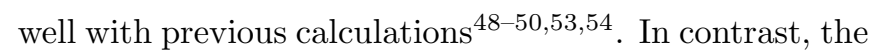
triangular hole pockets in the DFT-GGA band becomes to be a rounded hexagonal pocket in the $G W$ band as shown in Fig. 3 (b). So, flat sides of triangular pockets facing corners of hexagonal ones in the DFT-GGA Fermi surfaces protrude toward the $\Gamma$ point with $G W$ corrections. Then, the distance between the two band crossing points along the $\Gamma K$ line decreases with $G W$ corrections. This corresponds to the fact that the saddle point of the $G W$ band in the $M K$ line shifts up, as seen in Fig. 2, thus enabling one approach to the saddle point easier with slight hole doping.

\section{Noninteracting Susceptibility}

In order to investigate the implication of the change in the electronic structure on a formation of the charge density wave, we have calculated the real part of the noninteracting static susceptibility $\chi_{0}^{\prime}(\mathbf{q})^{50|53| 64]}[68$,

$\chi_{0}^{\prime}(\mathbf{q})=\sum_{n, n^{\prime}} \sum_{\mathbf{k}} \frac{f\left(\epsilon_{n \mathbf{k}}\right)-f\left(\epsilon_{n^{\prime} \mathbf{k}+\mathbf{q}}\right)}{\epsilon_{n \mathbf{k}}-\epsilon_{n^{\prime} \mathbf{k}+\mathbf{q}}}\left|\left\langle n \mathbf{k}\left|e^{i \mathbf{q} r}\right| n^{\prime} \mathbf{k}+\mathbf{q}\right\rangle\right|^{2}$,

where $\epsilon_{n \mathbf{k}}$ and $|n \mathbf{k}\rangle$ are the energy of the $n$-th band at the crystal momentum $\mathbf{k}$ and its corresponding Bloch state, and $f\left(\epsilon_{n \mathbf{k}}\right)$ is the Fermi-Dirac distribution function. Other quantum numbers such as spin are implicitly included in $n$. In principle, the matrix element 50166 is needed to calculate but it is known that the constant matrix approximation $[50|53| 67 \mid 68$, in which the matrix element is set to be unity, is good for the transition metal dichalcogenide ${ }^{68}$. The $k$-point grid used for the calculation is $300 \times 300 \times 1$.

Figure 4 shows the real part of the bare static susceptibility $\chi_{0}^{\prime}(\mathbf{q})$ of (a) DFT-GGA and (b) $G W$ calcu- 


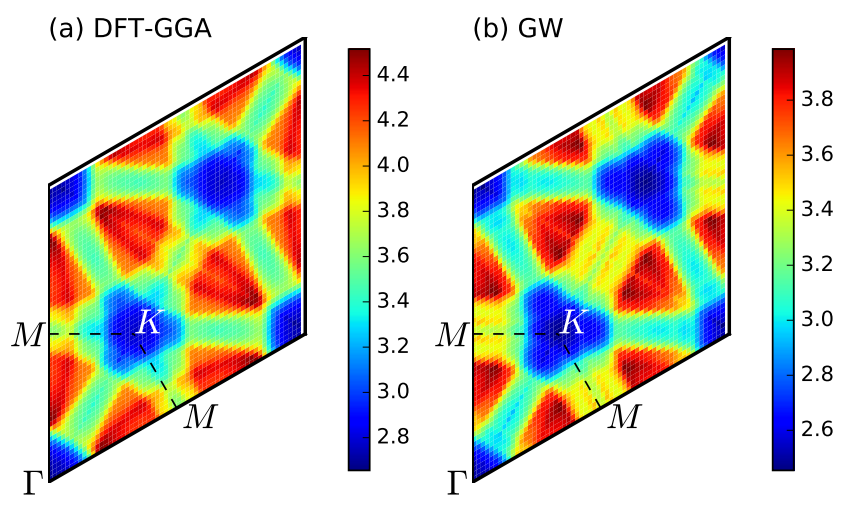

FIG. 4. (Color online) The real part of the noninteracting susceptibility $\chi_{0}^{\prime}(q)$ of (a) DFT-GGA and (b) $G W$ bands within the constant matrix approximation (in arbitrary units). The susceptibility is calculated at $k_{B} T=10 \mathrm{meV}$.

lations. We have checked that $\chi_{0}^{\prime}(\mathbf{q})$ of DFT-GGA has a broad maxima extending approximately from $2 / 5 \Gamma M$ to $4 / 5 \Gamma M$. Our calculation agrees well with other previous studies on the bulk structure $4 \sqrt{400}$ as well as the single layer ${ }^{53}$. With $G W$ approximation, a general shape of $\chi_{0}^{\prime}(\mathbf{q})$ is quite similar to that with DFT-GGA [Fig. 4 (b)] in spite of apparent differences between Fermi surfaces from the two calculation methods. We note that a
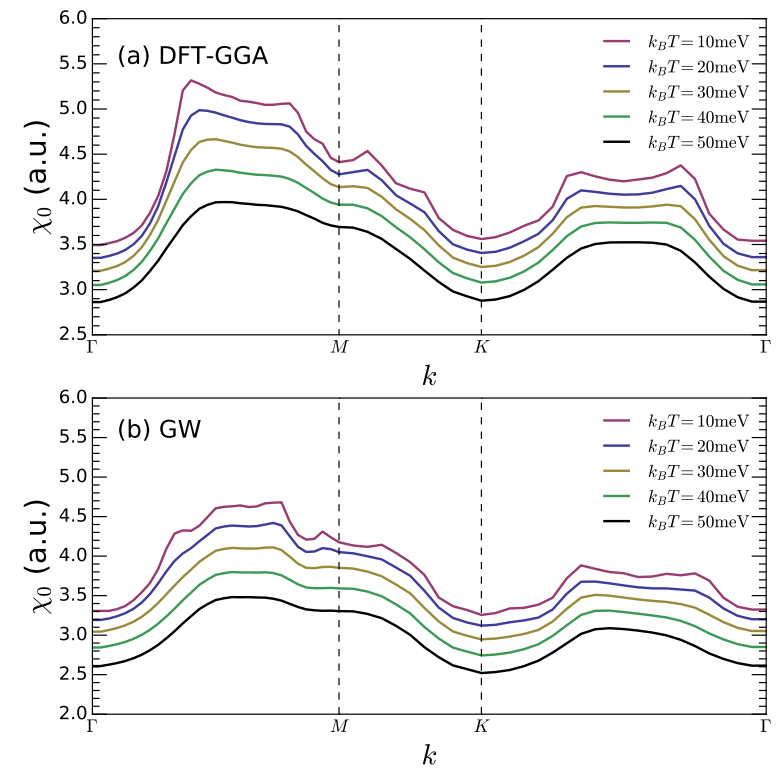

FIG. 5. (Color online) The real part of the noninteracting susceptibility $\chi_{0}(q)$ of (a) DFT-GGA and (b) $G W$ bands along the IBZ boundary $(\Gamma M K)$ for several temperatures $\left[k_{B} T=10\right.$ (purple), 20 (blue), 30 (yellow), 40 (green), and 50 (black) meV]. For clarify, the curves are shifted vertically by proper amounts.
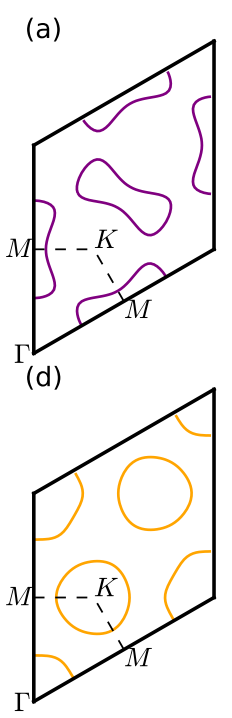

(b)
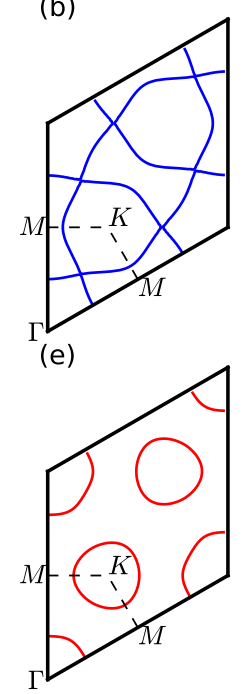

(c)

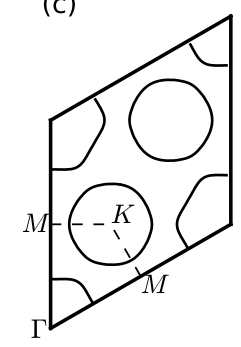

FIG. 6. (Color online) Fermi Surfaces (blue solid lines for the hole doped case and red for electron) of the single layer where the Fermi energy is rigidly shifted by $\delta \epsilon_{F}$ due to doping from the charge neutral one (black): (a) $\delta \epsilon_{F}=-150 \mathrm{meV}$, (b) $\delta \epsilon_{F}=-77 \mathrm{meV}$, (c) $\delta \epsilon_{F}=0 \mathrm{meV}$, (d) $\delta \epsilon_{F}=80 \mathrm{meV}$, and (e) $\delta \epsilon_{F}=150 \mathrm{meV}$.

peak is at $\left(\frac{1}{3} \vec{a}_{1}+\frac{1}{15} \vec{a}_{2}\right)$ point of IBZ not along the high symmetric line unlike the case with DFT-GGA.

In order to see the temperature dependence of $\chi_{0}^{\prime}(\mathbf{q})$, we have repeated $\chi_{0}^{\prime}(\mathbf{q})$ calculations by changing temperature $k_{B} T$. Figure 5 shows the bare susceptibility $\chi_{0}^{\prime}(\mathbf{q})$ along the IBZ boundary at several temperatures. At $k_{B} T \leq 10 \mathrm{meV}$, the maximum plateau of the DFTGGA susceptibility has a subpeak at $\mathbf{q} \approx 2 / 5 \Gamma M$. As temperature increases, this subpeak disappears and the broad plateau between $2 / 5 \Gamma M$ and $4 / 5 \Gamma M$ remains. For the $G W$ case, the trend is quite similar to those with DFT-GGA along the high symmetric lines of BZ.

\section{Doping Effects}

Next we consider the doping effect on the bare static susceptibility. The doping effect might be taken into account by rigidly shifting the Fermi energy. $\delta \epsilon_{F}$ denotes the Fermi energy shift with respect to the charge neutral level due to the doping effect. Figure 6 shows Fermi surfaces for two $p$-doped cases [(a) $\delta \epsilon_{F}=-150 \mathrm{meV}$, (b) $\delta \epsilon_{F}=-77 \mathrm{meV}$ ], the undoped case [(c) $\left.\delta \epsilon_{F}=0 \mathrm{meV}\right]$, and two $n$-doped cases [(d) $\delta \epsilon_{F}=80 \mathrm{meV}$, (e) $\delta \epsilon_{F}=150$ meV]. First we consider $p$-doped cases [Fig. 6(a) and (b)]. As seen in Fig. 2, the saddle point becomes closer to the Fermi level as the system is more $p$-doped. Thus, one can expect that the distance between the two hole pockets gets shorter. In particular, Fig. 6(b) displays the special case where the Fermi level touches the saddle point 
on the $\Gamma K$ line. In this case, two hole pockets (triangle and hexagon) are connected to each other. The Fermi surface in Fig. 6(b) is exactly the same one discussed in Ref. 51. So, a slight hole doping enable the system undergo CDW phase transition through the logarithmic divergence of the susceptibility at the CDW wave vector connecting the saddle points 51 . Being more $p$-doped, the topology of the Fermi surface is totally different from that of the undoped one. As shown in Fig. 6(a), triangular and hexagonal hole pockets are no longer observed, but there is only one rounded rectangular electron pocket, thus changing its carrier type.

In contrast, as shown in Figs. 6(d) and (e), the topology of the $n$-doped system does not change compared with the undoped case, except for the fact that the size of the two pockets is reduced.

Such a difference between Fermi surfaces of $p$-doped and $n$-doped cases leads to a qualitative change in the bare static susceptibility $\chi_{0}^{\prime}(\mathbf{q})$. For the $n$-doped cases where the topology of the Fermi surface does not change, the landscape of the noninteracting susceptibility $\chi_{0}^{\prime}(\mathbf{q})$ resembles that of the undoped system. See Fig. 7(c) and (d) for $\chi_{0}^{\prime}(\mathbf{q})$ of $\delta \epsilon_{F}=80 \mathrm{meV}$ and $150 \mathrm{meV}$, respectively. Compared with the undoped case, the minor difference is that the peak moves slightly toward $\Gamma M$ line, and the broad maximum from $3 / 5 \Gamma M$ to $4 / 5 \Gamma M$ is enhanced.

On the contrary, for the $p$-doped cases where the relative position of the saddle point is critical, the topological change in the Fermi surface gives rise to a qualitatively different susceptibility pattern. For low doping, i.e., $\delta \epsilon_{F}>-77 \mathrm{meV}$, the susceptibility pattern does not greatly deviate from that of the undoped system [See Fig. 7(a)]. However, when the system is more $p$ doped such that the Fermi energy is below the saddle point, quite a different pattern emerges. For example, see Fig. $7(\mathrm{~b})$ where $\delta \epsilon_{F}=-150 \mathrm{meV}$. As shown in the Fermi surface for the case of $\delta \epsilon_{F}=-77 \mathrm{meV}$, the peak of $\chi_{0}^{\prime}(\mathbf{q})$ for this case is at the $M$-point indicating its logarithmic divergence shown in Fig. 7 (a). One noticeable change for the case of further $p$-doping is that the largest peak lies neither at the $M$ point nor on the $\Gamma M$ line [Fig. 7 (b)]. Rather, the main peak is found on the $\Gamma K$ line for the case of $\delta \epsilon_{F}=-150 \mathrm{meV}$.

\section{E. Effect of Spin-Orbit Coupling}

So far the effect of spin-orbit coupling (SOC) has not been taken into account. Unlike the bulk $2 H$ structure where the combination of time-reversal and inversion symmetries prohibits band splitting due to SOC 69 , a single layer of the $2 H$ structure can be significantly affected by SOC, since there is no center of inversion symmetry. One might expect that a new feature can emerge on the Fermi surface, depending on whether the band splitting due to SOC is comparable to the energy of the saddle point with respect to the Fermi level.

For DFT calculations fully relativisitic pseudopoten-
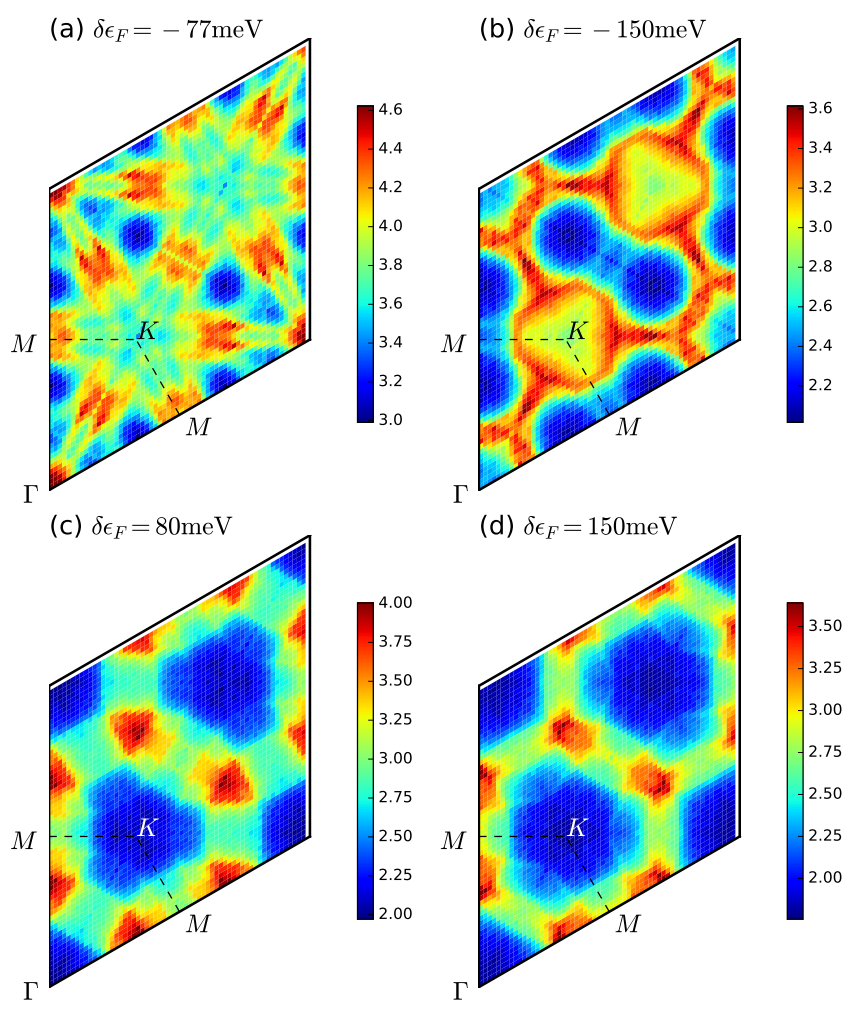

FIG. 7. (Color online) $\chi_{0}^{\prime}(\mathbf{q})$ of $p$-doped cases for $G W$ bands: (a) $\delta \epsilon_{F}=-77 \mathrm{meV}$ and (b) $\delta \epsilon_{F}=-150 \mathrm{meV}$. $\quad \chi_{0}^{\prime}(\mathbf{q})$ of $n$ doped cases for $G W$ bands: (c) $\delta \epsilon_{F}=80 \mathrm{meV}$ and (d) $\delta \epsilon_{F}=$ $150 \mathrm{meV}$.

tials are used. SOC are included on the final stage of the $G W$ approximation by adding DFT-SOC results since the effect of SOC to $G W$ results is marginal as shown in Ref. 70. We also note that a similar calculation method for the SOC effects has been used for explaining optical spectrum of a single layer $\mathrm{MoS}_{2}$ successfully 10111. Figure 8 shows the electronic band structure corrected by SOC around the Fermi energy from (a) DFT-GGA and (b) $G W$ schemes. As expected, the single band around the Fermi level is splitted to two bands for both cases. Note that the electronic band on the $\Gamma M$ line is not affected by SOC for the both cases. This can be understood by the fact that there is a mirror symmetry with respect to a plane perpendicular to the lattice vector $\mathbf{a}_{1}$ in Fig. 1(b). This mirror symmetry together with timereversal symmetry prohibits band splitting due to SOC along the $\Gamma M$ line.

With SOC corrections, we found that the general shape of energy bands are quite similar for the both calculation methods except the local Fermi surfaces shape near the saddle point. As shown in Fig. 9(a), SOC introduces two rounded triangular pockets around $K$ and two hexagons around $\Gamma$ to the Fermi surface of DFT-GGA, which are just two copies of the Fermi surface without SOC. In contrast, with $G W$ approximation, the two pockets along 

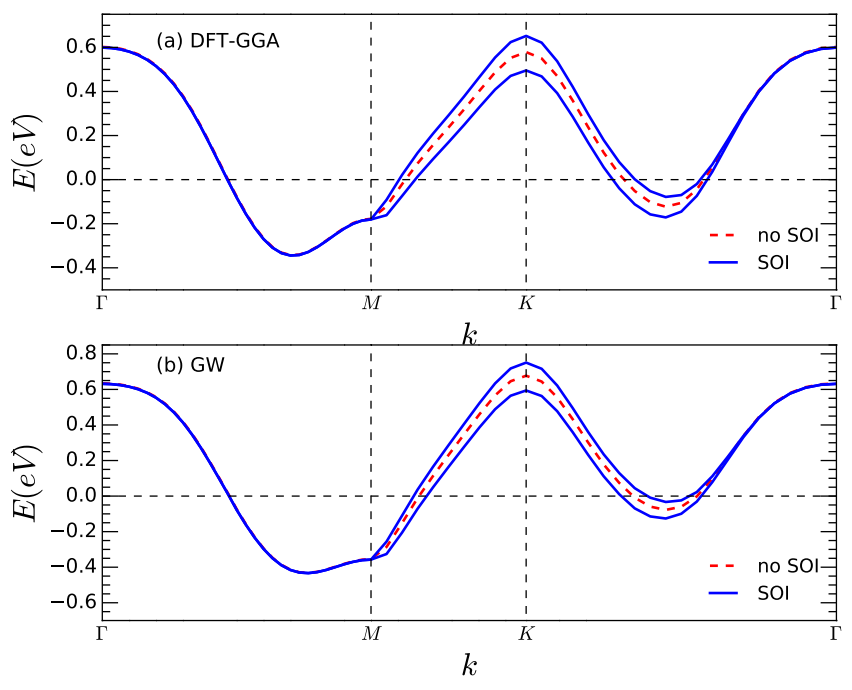

FIG. 8. (Color online) Partially occupied bands splitted by SOC along the IBZ boundary: (a) DFT-GGA and (b) $G W$ bands. Red dashed lines and blue solid ones indicate electronic bands without and with SOC, respectively.
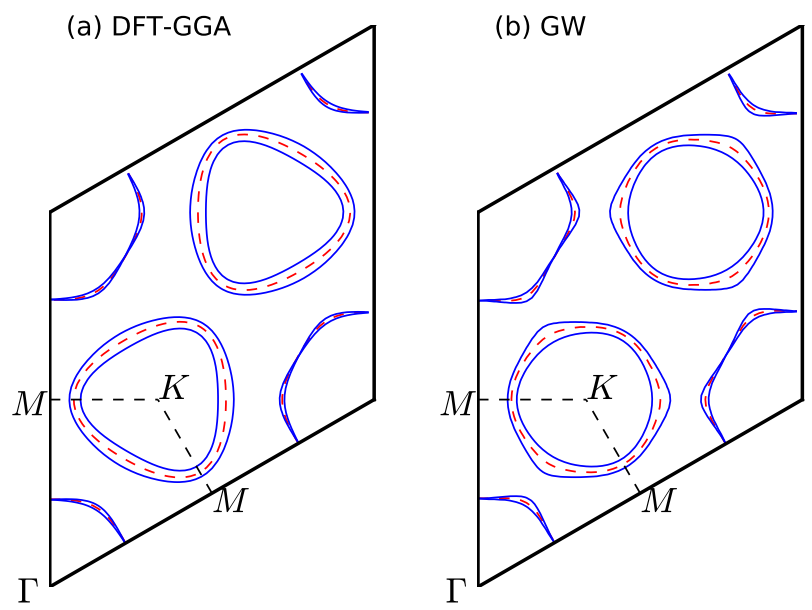

FIG. 9. (Color online) Fermi surfaces of electronic bands corrected by SOC: (a) DFT-GGA and (b) $G W$ bands. Red dashed lines and blue solid ones indicate Fermi surfaces without and with SOC, respectively.

the $\Gamma K$ line protrude toward each other further reducing the distance between the two pockets. So, from the $G W$ Fermi surface with SOC [Fig. 9 (b)], we expect that the small amount of $p$ doping can push the Fermi energy down to $34 \mathrm{meV}$ below the charge neutral point connecting two rectangular pockets through saddle points between them. This also ensures the logarithmic divergence of the susceptibility making CDW phase a possibility 51 .

We also emphasize that the Fermi surface of DFTGGA with SOC [Fig. 9(a)] is not a general result for a single layer of other metallic $2 H$-TMDs. For example, a single layer of $2 \mathrm{H}-\mathrm{TaSe}_{2}$ shows the Fermi surface similar to Fig. 9(b) except for that the orientation of the triangular pocket is inverted $\frac{69}{6}$. Thus, the Fermi surface of a single layer $2 H$-TMDs is determined by competition between the energy of the saddle point measured from the Fermi level and SOC-induced energy correction, which varies material by material.

We next consider the bare static susceptibility $\chi_{0}^{\prime}(\mathbf{q})$ for bands corrected by SOC. Special cares are needed when the constant matrix approximation is applied to Eq. (1) for the SOC-corrected band structure. For spindegenerate bands without SOC, the oscillator strength matrix element between different spin components is obviously zero. Thus, the spin degree of freedom, which is implicit in Eq. (1), just gives the factor two to $\chi_{0}^{\prime}(\mathbf{q})$. In contrast, the electronic bands corrected by SOC cannot be labelled by a definite spin quantum number over $\mathbf{k}$ space, but generally have a spin texture varying on $\mathbf{k}$ space. Thus it is likely that the interband matrix element of the oscillator strength might vanish for some points or regions of $\mathbf{k}$ space. It might not be correct to replace the interband matrix element of the oscillator strength just as unity for the whole $\mathbf{k}$ space without justification.

For the single layer of metallic TMDs, the bare susceptibility is mainly determined by two bands splitted by SOC around the Fermi level. It is known that these bands are composed primarily of $d_{z^{2}}, d_{x y}$, and $d_{x^{2}-y^{2}}$ orbitals of transition metal ${ }^{71}$ 75). In this effective subspace $\left\{d_{z^{2}}, d_{x y}, d_{x^{2}-y^{2}}\right\}$, the SOC term does not mix spin components. In other words, the two bands splitted by SOC have the opposite spin over $\mathbf{k}$ space. Therefore, interband components of the oscillator strength between the two bands might vanish, and the constant matrix approximation can be applied to intraband components. Note that this is the zeroth approximation, and this argument might be modified when $p$ orbitals of chalcogens and their SOC are involved. For detailed discussions on the effective tight-binding model of $d$ orbitals and the atomic SOC, see Appendix B and C

Figure 10 shows the bare susceptibility $\chi_{0}^{\prime}(\mathbf{q})$ calculated from DFT-GGA and $G W$ methods based on the above argument. The bare susceptibility of SOCcorrected bands looks similar to that of electronic bands without SOC in Fig. 4 except for that there are more substructures for the SOC-corrected bands. For example, the maximum plateau of DFT-GGA bands on the $\Gamma M$ line has more steps as shown in Fig. 10(a). For $G W$ bands, the bare susceptibility follows a similar variation with the case of DFT-GGA [Fig. 10(b)].

\section{DISCUSSION AND CONCLUSION}

Having established the quasiparticle energy bands and the Fermi surface of a monolayer $\mathrm{NbSe}_{2}$ using $G W$ approximation, now we discuss their implications on a possible CDW phase in the single layer on top of various substrates. Following previous discussions about ef- 


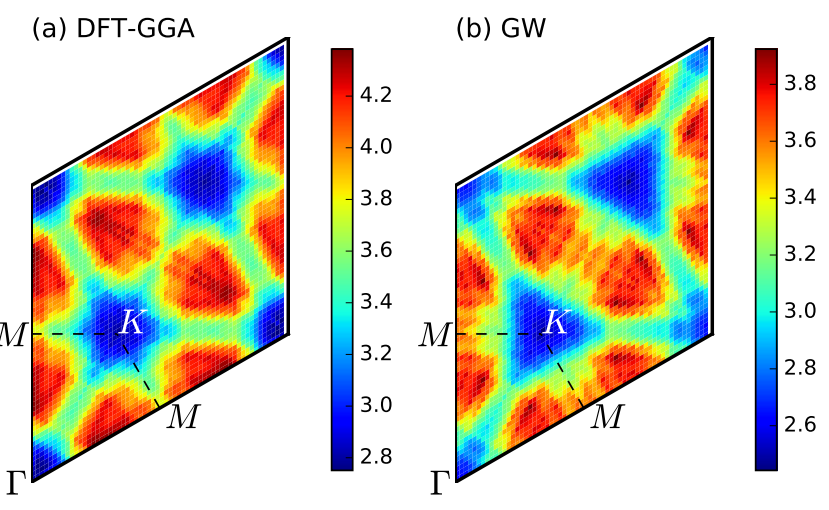

FIG. 10. (Color online) The real part of the non-interacting susceptibility $\chi_{0}^{\prime}(q)$ of SOC-corrected electronic bands from (a) DFT-GGA and (b) $G W$ methods.

fects of Coulomb interactions on the low energy bands of graphene on top of substrates $\frac{1318}{18}$, we consider alternations of the static screened Coulomb interaction in the momentum space $\frac{61}{63}, W_{\mathbf{G G}^{\prime}}=\epsilon_{\mathbf{G G}^{\prime}}^{-1}(\mathbf{q}) v\left(\mathbf{q}+\mathbf{G}^{\prime}\right)$ by approximately including environmental static dielectric screening $\left(\epsilon_{\text {env }}\right)$ in the dielectric matrix $\left(\epsilon_{\mathbf{G G}^{\prime}}\right)$ within the random phase approximation (RPA) such as,

$$
\epsilon_{\mathbf{G G}^{\prime}}(\mathbf{q}) \simeq \epsilon_{\mathrm{env}} \delta_{\mathbf{G G}^{\prime}}-v(\mathbf{q}+\mathbf{G}) \chi_{\mathbf{G G}^{\prime}}(\mathbf{q}),
$$

where $v(\mathbf{q}+\mathbf{G})=4 \pi /|\mathbf{q}+\mathbf{G}|^{2}$ is the bare Coulomb interactions, $\chi_{\mathbf{G G}^{\prime}}$ is the non-interacting polarizability, $\mathbf{q}$ is a $2 \mathrm{D}$ vector in the IBZ, and $\mathbf{G}$ is a $2 \mathrm{D}$ reciprocallattice vector. Here, $\epsilon_{\mathrm{env}} \equiv\left(\epsilon_{\mathrm{vac}}+\epsilon_{\mathrm{sub}}\right) / 2$ where $\epsilon_{\mathrm{vac}}=1$ is a dielectric constant of vacuum and $\epsilon_{\mathrm{sub}}$ is a static dielectric constant of substrate $13 / 18$. We consider two different substrates, the silicon oxide substrate used in Ref. 30 and the BLG/6H-SiC substrate in Ref. 31. For the former, $\epsilon_{\mathrm{env}}=2.45$ where $\epsilon_{\mathrm{sub}}=3.9$ for the silicon oxide ${ }^{76}$ so that, like graphene on top of $\mathrm{BN}, \mathrm{SiC}$ or quartz substrates 1318 , the weak substrate screening would not change the $G W$ band structure. Therefore, we expect that the proximity of saddle points near the Fermi level in our $G W$-SOC calculations may introduce the new type of CDW phase. For the latter, there are some ambiguities in using the approximation of Eq. 2 because the dielectric function of bilayer graphene has a strong $\mathbf{q}$ dependence $^{3}$ and because BLG on $6 H$-SiC(0001) surface is usually $n$-doped with electric field perpendicular to the plane ${ }^{77}, 79$. Considering a large lattice mismatch between $\mathrm{NbSe}_{2}$ and BLG and rotational disorders between

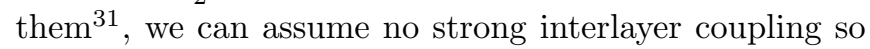
that the q-dependence of BLG dielectric function may be neglected. Taking into account of the doped BLG on top of $6 H$-SiC(0001) surface ${ }^{77+79}, \epsilon_{\text {sub }}$ becomes large for the in-plane polarization. Such a large substrate screening indicates that the Fermi surface and quasiparticles bands of the system follow those obtained from DFT-GGA calculations, thus favoring the bulk-like (strong electronphonon coupling induced) CDW phase transition 153 . We also note that a similar situation can occur in the bulk $\mathrm{NbSe}_{2}$. As shown in Fig. 14(e) of Appendix D our $G W$ bands calculation of bulk $2 H-\mathrm{NbSe}_{2}$ shows a quite similar Fermi surface shape near around $A$ - and $H$-points compared with those from a single layer DFT-GGA calculation. Such a similarity of Fermi surface shape is due to the relatively large screening effects of adjacent layers in the bulk. Although a large screening of the substrate could reduce the effects of strong Coulomb interaction, there may be a still considerable mismatch between the dispersion with a relatively large substrate screening and the DFT-GGA energy bands as shown in a previous study on the low energy quasiparticle energy bands of graphene 18 . So, we expect that the Fermi surface of the latter system could not entirely follow those from the mean-field calculation but that it could be a mixture between those two calculation schemes. So, subtle changes in the Fermi surface from the weak correction of $G W$ approximation could change the CDW periodicity different from the recent DFT-GGA calculation 53 .

The Fermi surface variation within the $G W$ approximation alone cannot explain the large discrepancy of $T_{\mathrm{CDW}}$ between the two experiments 30131 . Since a giant phonon softening should occur in the vicinity of $M$-point when the saddle points touch the Fermi energy ${ }^{51}$, this will enhance the transition temperature beyond the simple mean-field estimation but the more comprehensive studies to understand electron-phonon interaction with strong Coulomb interaction are need to understand this phenomena, that is beyond the scope of current work. A recent detailed calculation 80 for the single layer on top of graphene indicates a negligible interlayer interactionbetween them. Contrary to the experiment interpretation 31 , the detailed density of state analysis using DFTGGA calculation ${ }^{80}$ suggests a strong coupling mechanism for the CDW in the single layer limit but the origin of small energy gap at the Fermi energy is still not clear yet.

In conclusion, we have calculated quasiparticle band structures of a single layer $2 \mathrm{H}-\mathrm{NbSe}_{2}$ by using firstprinciples $G W$ calculation. We found that the width of a partially occupied band increases and its Fermi surface shape changes significantly compared with those obtained using DFT-GGA calculation method. The SOC changes the Fermi surfaces further and the resulting energy bands have the singular saddle points very close to the Fermi surface, enabling the system undergo CDW phase transition. Considering a relatively easy control of charge doping in $2 \mathrm{D}$ crystals, we think that the present system is particularly interesting in realizing dopingdependent phase transition. We also provide a simple tight-binding model with a basis of three $d$-orbitals of niobium that can be useful for many-body calculation of large hybrid systems involving $\mathrm{NbSe}_{2}$ and other layered systems. 


\section{ACKNOWLEDGMENTS}

We thank Antonio Castro Neto, Cheol-Hwan Park, Ting Cao and Steven Louie for discussion. Y.-W.S. was supported by the National Research Foundation of the Ministry of Science, ICT and Future Planning Grants of Korean government (QMMRC, No. R11-2008-05301002-0). Computations were supported by the Center for Advanced Computation of Korea Insitute for Advanced Study.

\section{Appendix A: Effects of semicore states on $G W$ approximations}

Here we compare band calculation results of the $G W$ approximation with and without including $\mathrm{Nb}$ semicore states in the pseudopotential. It has been investigated that $G W$ energy bands can be significantly changed by the inclusion of core states of the transition metal, for example, in II-VI semiconductors like CdS $\frac{58}{5}$ or bulk copper 59 . In these materials, usual DFT band structures rarely change by including core states whose binding energies are well separated from those of valence states. Despite a distinct separation between core and valence states, when core states have a large spatial overlap with valence states, core states can strongly interact with valence states when the exchange part of the $G W$ selfenergy is calculated. This strong interaction between core and valence states indeed leads to a significant modification on the $G W$ band structure $58 \mid 59$.
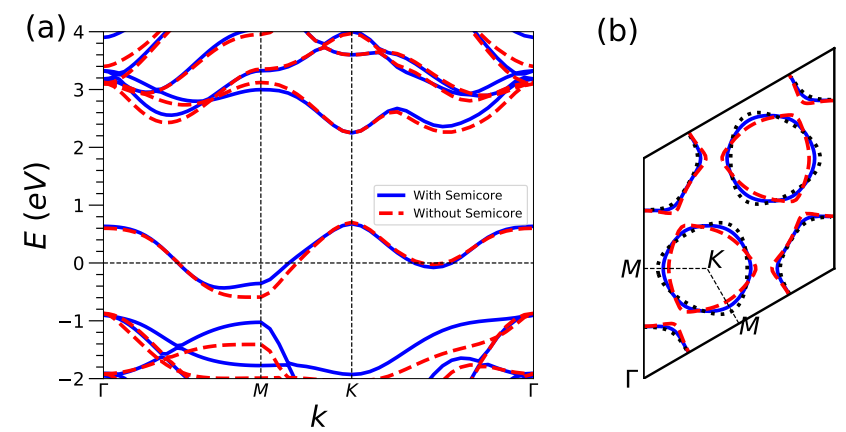

FIG. 11. (Color online) (a) $G W$ bands for a single layer $\mathrm{NbSe}_{2}$ with and without semicore levels of $4 s^{2} 4 p^{6}$ of $\mathrm{Nb}$ atom, which are denoted by blue solid lines and red dashed ones. (b) Fermi surfaces of $G W$ bands with semicore states (blue solid lines), $G W$ without semicore states (red dashed lines), and DFT-GGA bands (black dotted lines)

Regarding this effect, we have generated pseudopotentials of $\mathrm{Nb}$ atoms with the semicore $4 s^{2} 4 p^{6}$ electrons as valence ones and without them, and we have applied the $G W$ approximation to the two cases. Almost flat bands originating from $4 s$ and $4 p$ states are located at about $54.03 \mathrm{eV}$ and $30.39 \mathrm{eV}$, respectively, below the Fermi level, which are separated from $4 d$ bands around the Fermi level. All-electron calculation of the $\mathrm{Nb}$ atom reveals that radial wave functions of $4 s, 4 p$, and $4 d$ states mostly spread over the similar radial range, thereby implying that there is a strong interaction between core $(4 \mathrm{~s}$ and $4 p$ ) and valence $(4 d)$ states in the exchange interaction of the $G W$ approximation.

Figure 11(a) shows the resulting $G W$ band structures of the $\mathrm{NbSe}_{2}$ monolayer by using pseudopotentials with and without $4 s$ and $4 p$ semicore states. One distinguished feature induced by the inclusion of semicore states is the shape of the partially occupied band in the neighborhood of the symmetric point $M$. Without semicore states the minimum of the partially occupied band is located at $M$. It is in a sharp contrast to the fact that the partially occupied band has the energy minimum at the middle of the $\Gamma M$ line in the $G W$ approximation with semicore states. The energy difference of the two cases at $M$ is approximately $233 \mathrm{meV}$. Fermi surfaces of $G W$ bands with and without semicore states are shown in Fig. 11(b) together with the Fermi surface of DFT-GGA bands. Due to the energy shift around $M$ caused by semicore states, the crossing point of this band with the Fermi level on the $M K$ line moves toward the $M$ point, resulting in a rounded hexagonal Fermi surface around $M$. Without semicore states, the Fermi surface around $M$ looks like a triangular shape whose flat sides face the $M$ point, so the triangular pocket is distinguished from that of the DFTGGA calculation in terms of the orientation. Except for the energy correction around $M$, energies and shapes of partially occupied bands from the two calculations are almost the same.

When it comes to energy bands far from the Fermi level, it is first found that four lowest unoccupied bands originating from $4 d$ orbitals of $\mathrm{Nb}$ do not significantly change. In contrast higher unoccupied bands and fully occupied ones below the Fermi energy are shifted upwards overall.

\section{Appendix B: Atomic Spin-Orbit Coupling in monolayer $\mathrm{NbSe}_{2}$}

We here discuss the effect of SOC and the spin texture of the partially occupied band. Here we consider SOC as the atomic one that gives only on-site terms,

$$
\mathcal{H}_{\mathrm{so}}=\lambda_{\mathrm{TM}} \hat{\mathbf{S}} \cdot \sum_{m \in \mathrm{Nb}} \hat{\mathbf{L}}_{m}+\lambda_{\mathrm{CH}} \hat{\mathbf{S}} \cdot \sum_{m \in \mathrm{Se}} \hat{\mathbf{L}}_{m}
$$

where $\lambda_{\mathrm{TM}}$ and $\lambda_{\mathrm{CH}}$ are SOC constants for transition metal and chalcogen respectively, $\hat{\mathbf{S}}$ is the spin $1 / 2$ operator, and $\hat{\mathbf{L}}_{m}$ is the angular momentum operator of an atom $m$. First of all, for the $d$-orbital space, the SOC term at transition metal atom $m$ reads 


$$
\lambda_{\mathrm{TM}} \hat{\mathbf{S}} \cdot \hat{\mathbf{L}}_{m} \doteq \lambda_{\mathrm{TM}}\left[\begin{array}{cccccccccc}
0 & 0 & 0 & 0 & 0 & 0 & 0 & 0 & i \frac{\sqrt{3}}{2} & -\frac{\sqrt{3}}{2} \\
0 & 0 & i & 0 & 0 & 0 & 0 & 0 & \frac{1}{2} & -\frac{i}{2} \\
0 & -i & 0 & 0 & 0 & 0 & 0 & 0 & \frac{i}{2} & \frac{1}{2} \\
0 & 0 & 0 & 0 & \frac{i}{2} & -i \frac{\sqrt{3}}{2} & -\frac{1}{2} & -\frac{i}{2} & 0 & 0 \\
0 & 0 & 0 & -\frac{i}{2} & 0 & \frac{\sqrt{3}}{2} & \frac{i}{2} & -\frac{1}{2} & 0 & 0 \\
0 & 0 & 0 & i \frac{\sqrt{3}}{2} & \frac{\sqrt{3}}{2} & 0 & 0 & 0 & 0 & 0 \\
0 & 0 & 0 & -\frac{1}{2} & -\frac{i}{2} & 0 & 0 & -i & 0 & 0 \\
0 & 0 & 0 & \frac{i}{2} & -\frac{1}{2} & 0 & i & 0 & 0 & 0 \\
-i \frac{\sqrt{3}}{2} & \frac{1}{2} & -\frac{i}{2} & 0 & 0 & 0 & 0 & 0 & 0 & -\frac{i}{2} \\
-\frac{\sqrt{3}}{2} & \frac{i}{2} & \frac{1}{2} & 0 & 0 & 0 & 0 & 0 & \frac{i}{2} & 0
\end{array}\right],
$$

with the basis $\left\{\left|d_{z^{2}}, \uparrow\right\rangle,\left|d_{x y}, \uparrow\right\rangle,\left|d_{x^{2}-y^{2}}, \uparrow\right\rangle,\left|d_{y z}, \uparrow\right\rangle,\left|d_{x z}, \uparrow\right\rangle,\left|d_{z^{2}}, \downarrow\right\rangle,\left|d_{x y}, \downarrow\right\rangle,\left|d_{x^{2}-y^{2}}, \downarrow\right\rangle,\left|d_{y z} \downarrow\right\rangle,\left|d_{x z}, \downarrow\right\rangle\right\}$. Here $|\uparrow\rangle$ and $|\downarrow\rangle$ are spin eigenstates of the $\hat{S}_{z}$ operator.

Without SOC, it is shown that two subspaces of $d$ orbitals $\left\{d_{z^{2}}, d_{x y}, d_{x^{2}-y^{2}}\right\}$ and $\left\{d_{y z}, d_{x z}\right\}$ are not coupled in the group-theoretical construction of the $d$-orbital TB model 7172 . Equation (B2) tells us that SOC leads to mixing of the two subspaces $\left\{d_{z^{2}}, d_{x y}, d_{x^{2}-y^{2}}\right\}$ and $\left\{d_{y z}, d_{x z}\right\}$. Furthermore, $|\uparrow\rangle$ and $|\downarrow\rangle$ are no longer spin eigenstates, since $\left\langle\psi_{1}, \uparrow(\downarrow)\left|\hat{\mathbf{S}} \cdot \hat{\mathbf{L}}_{m}\right| \psi_{2}, \downarrow(\uparrow)\right\rangle \neq 0$ in which $\psi_{1} \in\left\{d_{z^{2}}, d_{x y}, d_{x^{2}-y^{2}}\right\}$ and $\psi_{2} \in\left\{d_{y z}, d_{x z}\right\}$.

When we consider the partially occupied band around the Fermi energy coming from $\left\{d_{z^{2}}, d_{x y}, d_{x^{2}-y^{2}}\right\}$, this band is well separated from the other four bands at least by $2 \mathrm{eV}$. Therefore, from the viewpoint of the perturbation theory, the mixing term of Eq. (B2) between $\left\{d_{z^{2}}, d_{x y}, d_{x^{2}-y^{2}}\right\}$ and $\left\{d_{y z}, d_{x z}\right\}$ might give a negligible contribution to the partially occupied band. In the subspace $\left\{d_{z^{2}}, d_{x y}, d_{x^{2}-y^{2}}\right\}$, the SOC term [Eq. B2 $]$ approximately reads

$$
\lambda_{\mathrm{TM}} \hat{\mathbf{S}} \cdot \hat{\mathbf{L}}_{m} \approx \lambda_{\mathrm{TM}}\left[\begin{array}{cccccc}
0 & 0 & 0 & 0 & 0 & 0 \\
0 & 0 & i & 0 & 0 & 0 \\
0 & -i & 0 & 0 & 0 & 0 \\
0 & 0 & 0 & 0 & 0 & 0 \\
0 & 0 & 0 & 0 & 0 & -i \\
0 & 0 & 0 & 0 & i & 0
\end{array}\right]
$$

which is written in the order of $\left\{\left|d_{z^{2}}, \uparrow\right\rangle,\left|d_{x y}, \uparrow\right\rangle,\left|d_{x^{2}-y^{2}}, \uparrow\right\rangle,\left|d_{z^{2}}, \downarrow\right\rangle,\left|d_{x y}, \downarrow\right\rangle,\left|d_{x^{2}-y^{2}}, \downarrow\right\rangle\right\}$ Clearly, Eq. (B3) has no mixing term between $|\uparrow\rangle$ and $|\downarrow\rangle$. Thus $|\uparrow\rangle$ and $|\downarrow\rangle$ can be regarded as good spin eigenstates for the partially occupied band in the effective $d$-orbital TB model discussed in Sec. C.

However, spin eigenstates for the real system might be modified, since there is the SOC term of $p$-orbital from chalcogens. The partially occupied band of our interest is approximately described in terms of three $d$ orbitals $\left\{d_{z^{2}}, d_{x y}, d_{x^{2}-y^{2}}\right\}$. However, it is an effective description, but the real band involves contribution from $p$ orbitals of chalcogens. In fact, according to the orbital-projected density of states calculation, the wavefunction of the partially occupied band contains $p$ orbitals of chalcogens by about $20 \%$.
The SOC term on a chalcogen $m$ is

$$
\lambda_{\mathrm{CH}} \hat{\mathbf{S}} \cdot \hat{\mathbf{L}}_{m} \approx \lambda_{\mathrm{CH}}\left[\begin{array}{cccccc}
0 & -\frac{i}{2} & 0 & 0 & 0 & \frac{1}{2} \\
\frac{i}{2} & 0 & 0 & 0 & 0 & -\frac{i}{2} \\
0 & 0 & 0 & -\frac{1}{2} & \frac{i}{2} & 0 \\
0 & 0 & -\frac{1}{2} & 0 & \frac{i}{2} & 0 \\
0 & 0 & -\frac{i}{2} & -\frac{i}{2} & 0 & 0 \\
\frac{1}{2} & \frac{i}{2} & 0 & 0 & 0 & 0
\end{array}\right],
$$

which is ordered in the basis of $\left\{\left|p_{x}, \uparrow\right\rangle,\left|p_{y}, \uparrow\right\rangle,\left|p_{z}, \uparrow\right\rangle,\left|p_{x}, \downarrow\right\rangle,\left|p_{y}, \downarrow\right\rangle,\left|p_{z}, \downarrow\right\rangle\right\} . \quad$ Two subspaces of $p$ orbitals $\left\{p_{x}, p_{y}\right\}$ and $\left\{p_{z}\right\}$ are mixed under SOC. Further, $|\uparrow\rangle$ and $|\downarrow\rangle$ are not exact spin eigenstates. Eigenstates of the total angular momentum operator $\hat{J}=\hat{L}+\hat{S}$ could be true ones since orbital and spin angular momenta are correlated via SOC. Considering contributions of $p$ orbitals, it might be expected that there is relatively small mixing between $|\uparrow\rangle$ and $|\downarrow\rangle$ components for bands splitted by SOC.

\section{Appendix C: Three-Band Tight-Binding Model}

In this Section, we construct a tight-binding (TB) model which closely reproduces $G W$ bands, especially the partially occupied band around the Fermi energy. The partially occupied band comes mostly from $d_{z^{2}}, d_{x y}$, and $d_{x^{2}-y^{2}}$ orbitals of transition metal71 75 . $p$ orbitals from chalcogen atoms gives the next contribution to the band. Although it is more precise to construct the tight-binding model by including the $p$ orbitals, we would like to have a minimal model capturing the main physics so that it could be used for other theoretical investigations. For this purpose, we start with the three-orbital TB model including only $d_{z^{2}}, d_{x y}$, and $d_{x^{2}-y^{2}}$ orbitals.

The form of the TB model and the number of independent parameters can be determined by the lattice symmetry. In particular, Ref. 71 provides a complete table of matrix components of the TB model of $d$ orbitals for hexagonal structure, which is relevant for TMDs. For detailed discussion on the group-theoretical construction of the TB model, see Refs. 72 and 73 . 
The effective TB model of $d$ orbitals has been applied to TMDs in the literature ${ }^{74 / 75}$. Recently, this model has been extensively used in order to fit DFT band structures for monolayers of group-VIB TMDs ${ }^{75}$. In Ref. 75, the authors have extended the three $d$-orbital TB model up to the third nearest neighbor (TNN) hoppings. Using the TNN TB model, they have successfully reproduced DFT electronic bands.

As mentioned in Refs. 74 and 75 , the $d$ - $d$ interactions in the effective TB model contain the direct hoppings between $d$ orbitals of transition metal and the indirect $d-d$ hoppings via $p$ orbitals of chalcogens. However, within this effective model, one cannot know how much contribution $p$ orbitals give to the energy band of our interest, which might be important for some aspects, for example, the effect of SOC.

Using the three-band TNN model of Ref. 75, we fit DFT-GGA and $G W$ bands on the IBZ boundary. For this, we first adopt the least-square fitting procedure, and finely tune parameters in order to fit the saddle point of the $M K$ line. Figure 12(a) shows energy bands of the three-band TNN TB model together with DFT-GGA bands. Following notations of Ref. 75, fitting parameters
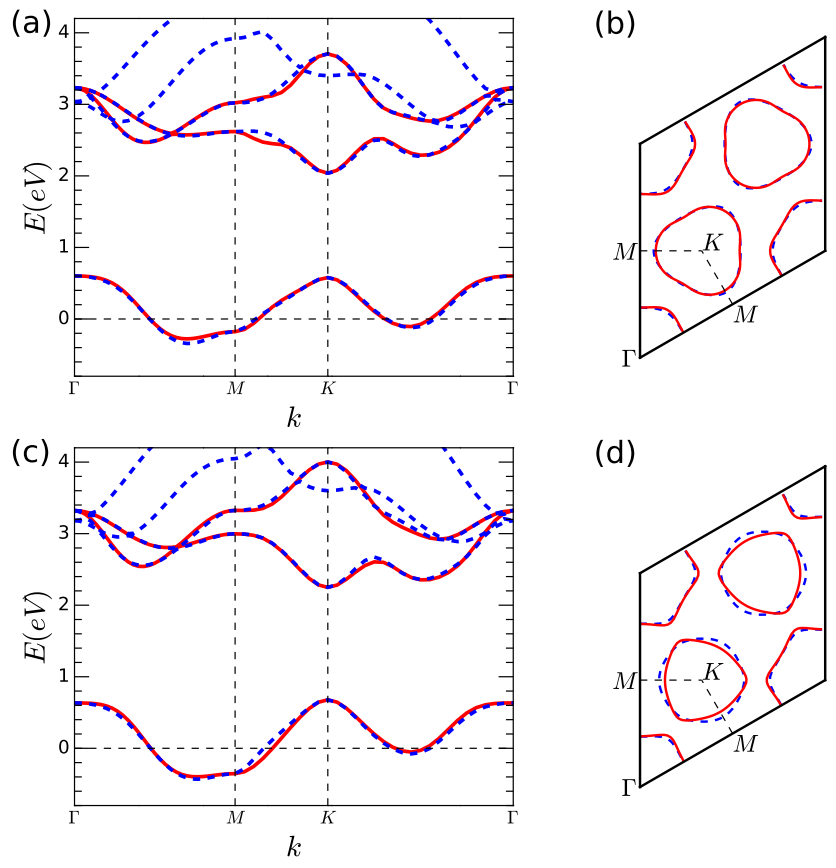

FIG. 12. (Color online) (a) Electronic bands from the DFTGGA calculation (blue dashed line) and ones from the threeband TNN TB model fit to the DFT-GGA bands (red solid line). (b) Fermi surfaces of electronic bands from the DFTGGA calculation (blue dashed line) and the TB model (red solid line).(c) Electronic bands from the $G W$ approximation (blue dashed line) and ones from the three-band TNN TB model fit to the $G W$ bands (red solid line). (d) Fermi surfaces of electronic bands from the $G W$ calculation (blue dashed line) and the TB model (red solid line).
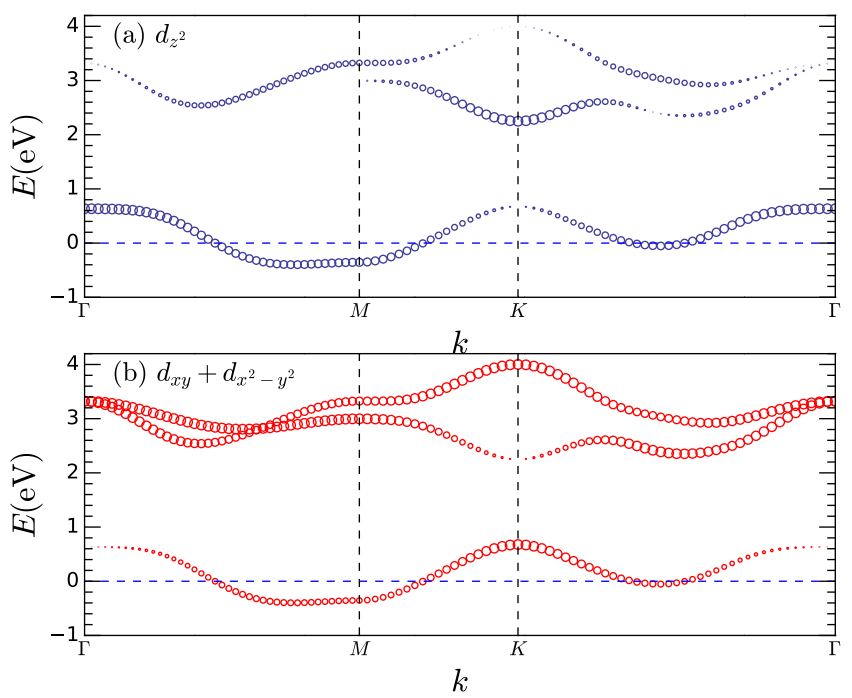

FIG. 13. (Color online) Orbital projected band structures from the three-band TNN TB model of the $G W$ bands: contributions from (a) the $d_{z^{2}}$ orbital (blue circle) and (b) $d_{x y}$ and $d_{x^{2}-y^{2}}$ orbitals (red circle). The size of open circle is proportional to contribution of the corresponding orbital. The Fermi energy (blue dashed line) is set to be zero.

of the TB model for DFT-GGA bands are $\epsilon_{1}=1.408$, $\epsilon_{2}=2.048, t_{0}=-0.128, t_{1}=0.115, t_{2}=-0.466, t_{11}=$ $0.115, t_{12}=0.122, t_{22}=0.036, r_{0}=0.025, r_{1}=0.194$, $r_{2}=-0.079, r_{11}=0.021, r_{12}=0.096, u_{0}=-0.031$, $u_{1}=-0.037, u_{2}=-0.002, u_{11}=0.258, u_{12}=-0.179$, and $u_{22}=-0.167$ in units of eV. The energy bands of the TB model are in a very good agreement with DFTGGA bands except for a small deviation at the energy minimum of the partially occupied band. As shown in Fig. 12(b), the fitted TB model well reproduces the Fermi surface of the DFT-GGA calculation.

We also construct the three-band TNN model of $G W$ bands, whose fitting parameters are $\epsilon_{1}=1.148, \epsilon_{2}=$ $2.379, t_{0}=-0.118, t_{1}=-0.386, t_{2}=-0.366, t_{11}=$ $0.167, t_{12}=0.243, t_{22}=-0.075, r_{0}=0.094, r_{1}=0.043$, $r_{2}=-0.152, r_{11}=0.055, r_{12}=-0.012, u_{0}=-0.061$, $u_{1}=-0.010, u_{2}=0.002, u_{11}=0.140, u_{12}=-0.077$, and $u_{22}=-0.014$ in units of eV. Energy bands reproduced by the TNN tight-binding model and $G W$ bands are shown in Fig. 12(c). The energy bands of the TB model agree well with those of the $G W$ calculation, but there is some deviation of the partially occupied band on the $M K$ line. While hexagonal pockets around $\Gamma$ nicely match, the triangular pocket of the TB model is less rounded than that of $G W$ bands. This is due to the small mismatch of the two bands on the $M K$ line in Fig. 12 (c).

Figure 13 displays orbital-projected bands calculated from the three-orbital TNN TB model of the $G W$ bands. $d_{z^{2}}$ orbital is dominant for the lowest band around the Fermi level, while $d_{x y}$ and $d_{x^{2}-y^{2}}$ orbitals give main con- 


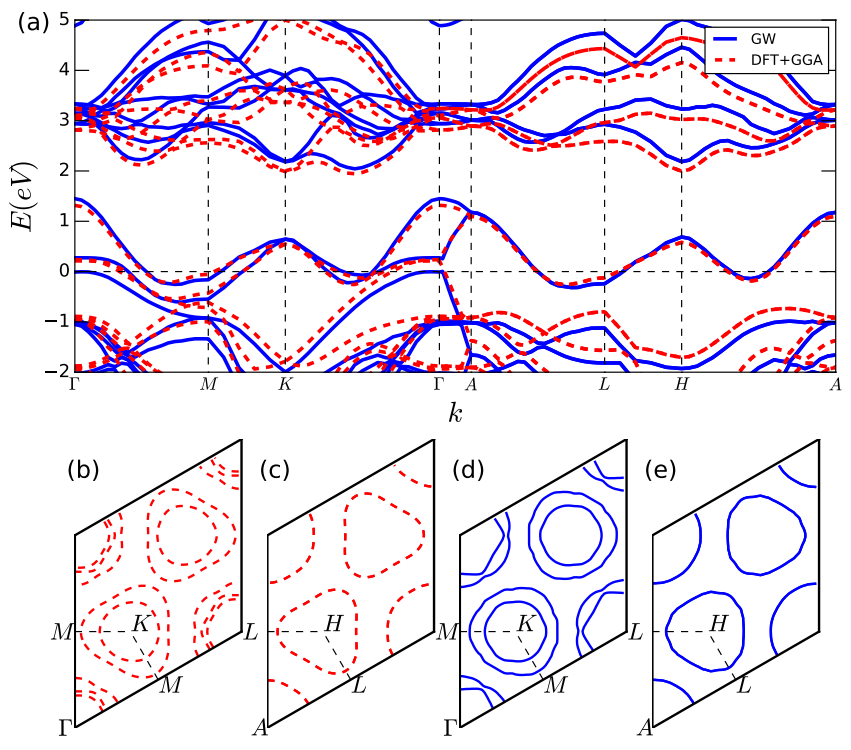

FIG. 14. (Color online) (a) Energy bands of the bulk $2 H$ $\mathrm{NbSe}_{2}$ along symmetric lines of the IBZ. Red dashed lines and blue solid lines correspond to DFT-GGA and $G W$ electronic bands, respectively. $(b)$ and $(c)$ are Fermi surfaces of DFT-GGA bands on $\Gamma M K$ and $A L H$ planes of the IBZ respectively. Similarly $(d)$ and $(e)$ shows Fermi surfaces of $G W$ bands on $\Gamma M K$ and $A L H$ planes, respectively.

tributions to the other two unoccupied bands.

\section{Appendix D: $G W$ bands of bulk $2 H-\mathrm{NbSe}_{2}$}

We have extended DFT-GGA and $G W$ calculations to energy bands of the bulk $2 H-\mathrm{NbSe}_{2}$ by using the same pseudo-potential including semi-core states. For detailed calculations we have used the plane-wave basis set with a cutoff of $55 \mathrm{Ry}$, a $20 \times 20 \times 5 \mathrm{k}$-point grid, and a smearing temperature $k_{B} \tau=0.005 \mathrm{Ry} 60$. The $G W$ bands of the bulk $2 \mathrm{H}-\mathrm{NbSe}_{2}$ is calculated within the level of the $G_{0} W_{0}$ approximation $\frac{63}{6 y}$ including about 400 unoccupied bands, which are up to 10 Ry above the Fermi energy.

Figure 14(a) shows DFT-GGA and $G W$ electronic bands of the bulk $2 H-\mathrm{NbSe}_{2}$ along symmetric lines of the IBZ, which are denoted by red dashed lines and blue solid ones respectively. Their corresponding Fermi surfaces are depicted in Fig. 14(b)-(d). Figure 14(b) and (c) show Fermi surfaces of DFT-GGA bands on the $\Gamma M K$ and $A L H$ planes, respectively, while Fig. 14 (d) and (e) are Fermi surfaces of $G W$ bands on $\Gamma M K$ and $A L H$ planes respectively. As discussed in Sec. IV] the Fermi surface of the bulk $2 H-\mathrm{NbSe}_{2} G W$ bands on the $A L H$ plane is similar to that of the single-layer DFT-GGA bands on the $\Gamma M K$ plane. In particular the Fermi surface around $H$ is a rounded triangular pocket whose flat lines face the symmetric point $A$, but not a rounded hexagonal pocket as seen in the Fermi surface of the monolayer $G W$ calculation. The relative large screening effect of neighboring layers could lead to this similarity between the Fermi surface of bulk $G W$ bands and that of monolayer DFT-GGA bands.
* Email: sejoong@ust.ac.kr

$\dagger$ Email: hand@kias.re.kr

${ }^{1}$ K. S. Novoselov, D. Jiang, F. Schedin, T. J. Booth, V. V. Khotkevich, S. V. Morozov, and A. K. Geim, Proc. Natl. Acad. Sci. USA 102, 10451 (2005).

2 A. H. Castro Neto, F. Guinea, N. M. R. Peres, K. S. Novoselov, and A. K. Geim, Rev. Mod. Phys. 81, 109 (2009).

3 S. Das Sarma, S. Adam, E. H. Hwang, and E. Rossi, Rev. Mod. Phys. 83, 407 (2011).

${ }^{4}$ Q. H. Wang, K. Kalantar-Zadeh, A. Kis, J. N. Coleman, and M. S. Strano, Nat. Nano. 7, 699 (2012).

5 V. N. Kotov, B. Uchoa, V. M. Pereira, F. Guinea, and A. H. Castro Neto, Rev. Mod. Phys. 84, 1067 (2012).

6 A. K. Geim and I. V. Grigorieva, Nature 499, 419 (2013).

7 H.-P. Komsa and A. V. Krasheninnikov, Phys. Rev. B 86, 241201 (2012).

8 A. Ramasubramaniam, Phys. Rev. B 86, 115409 (2012).

9 T. Cheiwchanchamnangij and W. R. L. Lambrecht, Phys.
Rev. B 85, 205302 (2012).

10 D. Y. Qiu, F. H. da Jornada, and S. G. Louie, Phys. Rev. Lett. 111, 216805 (2013).

11 M. M. Ugeda, A. J. Bradley, S.-F. Shi, F. H. da Jornada, Y. Zhang, D. Y. Qiu, W. Ruan, S.-K. Mo, Z. Hussain, Z.X. Shen, F. Wang, S. G. Louie, and M. F. Crommie, Nat. Mater. 13, 1091 (2014).

12 P. E. Trevisanutto, C. Giorgetti, L. Reining, M. Ladisa, and V. Olevano, Phys. Rev. Lett. 101, 226405 (2008).

13 C.-H. Park, F. Giustino, C. D. Spataru, M. L. Cohen, and S. G. Louie, Phys. Rev. Lett. 102, 076803 (2009).

14 A. Bostwick, F. Speck, T. Seyller, K. Horn, M. Polini, R. Asgari, A. H. MacDonald, and E. Rotenberg, Science 328, 999 (2010).

15 D. C. Elias, R. V. Gorbachev, A. S. Mayorov, S. V. Morozov, A. A. Zhukov, P. Blake, L. A. Ponomarenko, I. V. Grigorieva, K. S. Novoselov, F. Guinea, and A. K. Geim, Nat. Phys. 7, 701 (2011).

16 D. A. Siegel, W. Regan, A. V. Fedorov, A. Zettl, and 
A. Lanzara, Phys. Rev. Lett. 110, 146802 (2013).

17 J. Lischner, D. Vigil-Fowler, and S. G. Louie, Phys. Rev. Lett. 110, 146801 (2013).

18 C. Hwang, D. A. Siegel, S.-K. Mo, W. Regan, A. Ismach, Y. Zhang, A. Zettl, and A. Lanzara, Sci. Rep. 2, 590 (2012).

19 J. T. Ye, Y. J. Zhang, R. Akashi, M. S. Bahramy, R. Arita, and Y. Iwasa, Science 338, 1193 (2012).

${ }^{20}$ Y. Yu, F. Yang, X. F. Lu, Y. J. Yan, Y.-H. Cho, L. Ma, X. Niu, S. Kim, Y.-W. Son, D. Feng, S. Li, S.-W. Cheong, X. H. Chen, and Y. Zhang, Nat. Nano. 10, 270 (2015).

${ }^{21}$ K. Sugawara, Y. Nakata, R. Shimizu, P. Han, T. Hitosugi, T. Sato, and T. Takahashi, ACS Nano 10, 1341 (2015).

${ }^{22}$ P. Chen, Y.-H. Chan, X.-Y. Fang, Y. Zhang, M. Y. Chou, S.-K. Mo, Z. Hussain, A.-V. Fedorov, and T.-C. Chiang, Nat. Comm. 6, 8943 (2015).

23 J.-P. Peng, J.-Q. Guan, H.-M. Zhang, C.-L. Song, L. Wang, K. He, Q.-K. Xue, and X.-C. Ma, Phys. Rev. B 91, 121113 (2015).

24 Y. Saito, Y. Kasahara, J. T. Ye, Y. Iwasa, and T. Nojima, Science 350, 409 (2015).

25 Y. Saito, Y. Nakamura, M. S. Bahramy, Y. Kohama, J. T. Ye, Y. Kasahara, Y. Nakagawa, M. Onga, M. Tokunaga, T. Nojima, and Y. Y. adn Y. Iwasa, Nat. Phys. 12, 144 (2016).

${ }^{26}$ L. J. Li, E. C. T. O'Farrell, K. P. Loh, G. Eda, B. Özyilmaz, and A. H. Castro-Neto, Nature 529, 185 (2016).

27 X. Xi, Z. Wang, W. Zhao, J.-H. Park, K. T. Law, H. Berger, L. Forró, J. Shen, and K. F. Mak, Nat. Phys. 12, 139 (2016).

28 A. W. Tsen, B. Hunt, Y. D. Kim, Z. J. Yuan, S. Jia, R. J. Cava, J. Hone, P. Kim, C. R. Dean, and A. N. Pasupathy, Nat. Phys. 12, 208 (2016).

29 Y. Cao, A. Mishchenko, G. L. Yu, E. Khestanova, A. P. Rooney, E. Prestat, A. V. Kretinin, P. Blake, M. B. Shalom, C. Woods, J. Chapman, G. Balakrishnan, I. V. Grigorieva, K. S. Novoselov, B. A. Piot, M. Potemski, K. Watanabe, T. Taniguchi, S. J. Haigh, A. K. Geim, and R. V. Gorbachev, Nano Lett. 15, 4914 (2015).

30 X. Xi, L. Zhao, Z. Wang, H. Berger, L. Forró, J. Shan, and K. F. Mak, Nat. Nano. 10, 765 (2015).

31 M. M. Ugeda, A. J. Bradley, Y. Zhang, S. Onishi, Y. Chen, W. Ruan, C. Ojeda-Aristizabal, H. Ryu, M. T. Edmonds, H.-Z. Tsai, A. Riss, S.-K. Mo, D. Lee, A. Zettl, Z. Hussain, Z.-X. Shen, and M. F. Crommie, Nat Phys 12, 92 (2016).

32 J. A. Wilson, F. J. D. Salvo, and S. Mahajan, Adv. Phys. 24, 117 (1975).

33 K. Rossnagel, J. Phys.: Cond. Matter 23, 213001 (2011).

34 E. Revolinsky, E. P. Lautenschlager, and C. H. Armitage, Solid State Commun. 1, 59 (1963).

35 E. Revolinsky, G. A. Spiering, and D. J. Beerntsen, J. Phys. Chem. Solids 26, 1029 (1965).

36 J. A. Wilson, F. J. Di Salvo, and S. Mahajan, Phys. Rev. Lett. 32, 882 (1974).

37 T. Straub, T. Finteis, R. Claessen, P. Steiner, S. Hüfner, P. Blaha, C. S. Oglesby, and E. Bucher, Phys. Rev. Lett. 82, 4504 (1999).

38 D. W. Shen, Y. Zhang, L. X. Yang, J. Wei, H. W. Ou, J. K. Dong, B. P. Xie, C. He, J. F. Zhao, B. Zhou, M. Arita, K. Shimada, H. Namatame, M. Taniguchi, J. Shi, and D. L. Feng, Phys. Rev. Lett. 101, 226406 (2008).

39 S. V. Borisenko, A. A. Kordyuk, V. B. Zabolotnyy, D. S. Inosov, D. Evtushinsky, B. Büchner, A. N. Yaresko,
A. Varykhalov, R. Follath, W. Eberhardt, L. Patthey, and H. Berger, Phys. Rev. Lett. 102, 166402 (2009).

40 C. M. Varma and A. L. Simons, Phys. Rev. Lett. 51, 138 (1983).

41 T. Valla, A. V. Fedorov, P. D. Johnson, P.-A. Glans, C. McGuinness, K. E. Smith, E. Y. Andrei, and H. Berger, Phys. Rev. Lett. 92, 086401 (2004).

42 F. Weber, S. Rosenkranz, J.-P. Castellan, R. Osborn, R. Hott, R. Heid, K.-P. Bohnen, T. Egami, A. H. Said, and D. Reznik, Phys. Rev. Lett. 107, 107403 (2011).

43 D. J. Rahn, S. Hellmann, M. Kalläne, C. Sohrt, T. K. Kim, L. Kipp, and K. Rossnagel, Phys. Rev. B 85, 224532 (2012).

44 A. Soumyanarayanan, M. M. Yee, Y. He, J. van Wezel, D. J. Rahn, K. Rossnagel, E. W. Hudson, M. R. Norman, and J. E. Hoffman, Proc. Natl. Acad. Sci. USA 110, 1623 (2013).

45 C. J. Arguello, E. P. Rosenthal, E. F. Andrade, W. Jin, P. C. Yeh, N. Zaki, S. Jia, R. J. Cava, R. M. Fernandes, A. J. Millis, T. Valla, R. M. Osgood, and A. N. Pasupathy, Phys. Rev. Lett. 114, 037001 (2015).

46 R. F. Frindt, Phys. Rev. Lett. 28, 299 (1972).

47 N. E. Staley, J. Wu, P. Eklund, Y. Liu, L. Li, and Z. Xu, Phys. Rev. B 80, 184505 (2009).

48 M. D. Johannes and I. I. Mazin, Phys. Rev. B 77, 165135 (2008)

49 N. J. Doran, D. Titterington, B. Ricco, M. Schreiber, and G. Wexler, J. Phys. C: Solid State Phys. 11, 699 (1978).

50 M. D. Johannes, I. I. Mazin, and C. A. Howells, Phys. Rev. B 73, 205102 (2006).

51 T. M. Rice and G. K. Scott, Phys. Rev. Lett. 35, 120 (1975).

52 T. Kiss, T. Yokoya, A. Chainani, S. Shin, T. Hanguri, M. Nohara, and H. Takagi, Nat. Phys. 3, 720 (2007).

53 M. Calandra, I. I. Mazin, and F. Mauri, Phys. Rev. B 80, 241108 (2009).

54 S. Lebègue and O. Eriksson, Phys. Rev. B 79, 115409 (2009).

55 J. P. Perdew, K. Burke, and M. Ernzerhof, Phys. Rev. Lett. 77, 3865 (1996).

56 N. Troullier and J. L. Martins, Phys. Rev. B 43, 1993 (1991).

57 S. G. Louie, S. Froyen, and M. L. Cohen, Phys. Rev. B 26, 1738 (1982).

58 M. Rohlfing, P. Krüger, and J. Pollmann, Phys. Rev. Lett. 75, 3489 (1995).

59 A. Marini, G. Onida, and R. Del Sole, Phys. Rev. Lett. 88, 016403 (2001).

60 P. Giannozzi, S. Baroni, N. Bonini, M. Calandra, R. Car, C. Cavazzoni, D. Ceresoli, G. L. Chiarotti, M. Cococcioni, I. Dabo, A. D. Corso, S. de Gironcoli, S. Fabris, G. Fratesi, R. Gebauer, U. Gerstmann, C. Gougoussis, A. Kokalj, M. Lazzeri, L. Martin-Samos, N. Marzari, F. Mauri, R. Mazzarello, S. Paolini, A. Pasquarello, L. Paulatto, C. Sbraccia, S. Scandolo, G. Sclauzero, A. P. Seitsonen, A. Smogunov, P. Umari, and R. M. Wentzcovitch, J. of Phys.: Condens. Mat. 21, 395502 (2009).

61 L. Hedin, Phys. Rev. 139, A796 (1965).

${ }^{62}$ M. S. Hybertsen and S. G. Louie, Phys. Rev. B 34, 5390 (1986).

63 J. Deslippe, G. Samsonidze, D. A. Strubbe, M. Jain, M. L. Cohen, and S. G. Louie, Comput. Phys. Commun. 183, 1269 (2012).

64 J. F. Cooke, H. L. Davis, and M. Mostoller, Phys. Rev. B 
9, 2485 (1974).

65 J. Rath and A. J. Freeman, Phys. Rev. B 11, 2109 (1975).

${ }^{66}$ R. P. Gupta and A. J. Freeman, Phys. Rev. B 13, 4376 (1976).

67 H. W. Myron and A. J. Freeman, Phys. Rev. B 11, 2735 (1975).

${ }^{68}$ H. W. Myron, J. Rath, and A. J. Freeman, Phys. Rev. B 15, 885 (1977).

69 Y. Ge and A. Y. Liu, Phys. Rev. B 86, 104101 (2012).

${ }^{70}$ O. V. Yazyev, E. Kioupakis, J. E. Moore, and S. G. Louie, Phys. Rev. B 85, 161101 (2012).

71 M. Miasek, Phys. Rev. 107, 92 (1957).

72 R. F. Egorov, B. I. Reser, and V. P. Shirokovskii, Phys. Status Solidi B 26, 391 (1968).

73 V. N. Kuznetsov and A. N. Men, Phys. Status Solidi B 85, 95 (1978).
74 L. F. Mattheiss, Phys. Rev. Lett. 30, 784 (1973).

75 G.-B. Liu, W.-Y. Shan, Y. Yao, W. Yao, and D. Xiao, Phys. Rev. B 88, 085433 (2013).

76 P. R. Gray, P. J. Hurst, S. H. Lewis, and R. G. Meyer, Analysis and Design of Analog Integrated Circuits (Wiley, New York, 1984).

77 T. Ohta, A. Bostwick, J. L. McChesney, T. Seyller, K. Horn, and E. Rotenberg, Phys. Rev. Lett. 98, 206802 (2007).

78 S. Y. Zhou, G.-H. Gweon, A. V. Fedorov, P. N. First, W. A. de Heer, D.-H. Lee, F. Guinea, A. H. C. Neto, and A. Lanzara, Nat. Mat. 6, 770 (2007).

79 S. Kim, J. Ihm, H. J. Choi, and Y.-W. Son, Solid State Comm. 175-176, 83 (2013).

80 J. A. Silva-Guillén, P. Ordejón, F. Guinea, and E. Canadell, 2D Mater. 3, 035028 (2016). 